\title{
ANTHOLOGIA SANSCRITICA
}

\section{GLOSSARIO INSTRVCTA.}

\author{
IN VSVM SCHOLARVM
}

EDIDIT

\section{CHRISTIANVS LASSEN}

PHIL. DR. LITERARVM INDICARVM IN VNIVERSITA'TE BONNENSI PROFESSOR P. O. CET. CEr.

DENYO ADORNATIT

IOANNES GILDEMEISTER

THEOL. ET PHIL. DR. LITFRARVM ORIENTALIVM IN EADEM VNIVERSITATE PROFESSOR P. o.

EDITIO ALTERA

NOVIS CVRIS RETRACTATA.

BONNAE AD RHENVM

APVD ADOLPHVM MARCVM

Moccchximl 
